\title{
Role of Mucosal Colonic Biopsy in Patients with Chronic Unexplained Diarrhoea who their Colonoscopy is Normal
}

Dalia M Badary ${ }^{1^{*}}$ and Mohamed Z Hafez ${ }^{2}$

${ }^{1}$ Pathology Department, Assuit University, Al-Gamaa Street, Egypt

${ }^{2}$ Internal Medicine Department, Aswan University, Egypt

*Corresponding author: Dalia M Badary, Pathology Department, Assuit University, Al-Gamaa Street, Egypt, Tel: 00201066189050; E-mail: hamasat82@yahoo.com

Received date: January 31, 2017; Accepted date: February 6, 2017; Published date: February 13, 2017

Copyright: ( 2017 Badary DM, et al. This is an open-access article distributed under the terms of the Creative Commons Attribution License, which permits unrestricted use, distribution, and reproduction in any medium, provided the original author and source are credited.

\begin{abstract}
Objective: We sought to determine the importance of histopathological assessment in patients with chronic diarrhoea who had macroscopically normal colonic endoscopies.

Methods: Of 300 consecutive patients undergoing colonoscopy by one endoscopist during a five year period, biopsies were taken in 200 cases of unexplained diarrhoea of at least 6 weeks and their colorectal mucosa appeared macroscopically normal. All biopsies were reviewed by one pathologist.

Results: Of the 200 patients enrolled, $36(18 \%)$ cases were classified as unremarkable histopathological diagnosis and $164(82 \%)$ cases showed histopathological changes: 121 (73.78\%) non-specific inflammation, 19 $(11.58 \%)$ ulcerative colitis, $11(6.7 \%)$ collagenous colitis, $7(4.26 \%)$ lymphocytic colitis, and 6 (3.65\%) bilharzial colitis.

Conclusion: We conclude that the role of biopsies in chronic diarrhoea patients with grossly normal colon at endoscopy is high as yielding a histological diagnosis in $21.5 \%$ of patients is so benefit to them as they may had modified the therapy received after identification of a specific cause as collagenous colitis, lymphocytic colitis, ulcerative colitis and bilharzial colitis. So Patients should be subject to colonoscopy and biopsy to aid specific diagnoses.
\end{abstract}

Keywords: Colonoscopy; Diarrhoea; Colitis; Collagen; Lymphocytes; Bilharziasis

\section{Introduction}

Chronic diarrhoea is one of the most common conditions presented to a gastroenterologist. It may be defined as the passage of abnormally liquid or unformed stools at an increased frequency as stool weight $\geq$ $200 \mathrm{~g} /$ day and duration is $\geq 4$ weeks. It has varied etiology like infections, inflammatory bowel disease, neoplasia, diverticular disease, endocrine-metabolic, functional, drug induced or as a side effect of radiation therapy [1]. Thus the diagnosis of chronic diarrhoea is complex [2].

Colonscopy is one of the most important tools for investigation of colorectal diseases as it has been used to screen large bowel to identify early lesions in many risk groups and to investigate patients with various complains as abdominal pain, changes in bowel habits, gastrointestinal bleeding, chronic diarrhoea and abdominal masses [3].

However, some patients with chronic diarrhoea reveal a normal colonoscopy, their biopsies may provide the information required to establish a perfect diagnosis of the main responsible cause of this diarrhoea and prescribe sufficient treatment [4].

According to the Royal College of Pathologists, the histopathological findings of colonoscopic biopsies only have predictive and clinical value in patients with persistent diarrhoea without bleeding [4]. The guidelines set forth the Quality and Safety
Indicators for Endoscopy issued by the British Society of Gastroenterology in association with the Joint Advisory Group in Gastrointestinal Endoscopy, recommend performing biopsies in all patients suffering from persistent diarrhoea [5]. Although there are many indications for conducting colonoscopies, we considered in this study only the cases of chronic diarrhoea undergoing colonoscopy which was normal and doing subsequent biopsy hoping that these biopsies help in reaching the main cause of the diarrhoea and trying to put management guidelines of cases with chronic diarrhoea.

This study was designed to detect the rates of histological diagnoses made from seemingly normal mucosa in our locality and to determine the necessity of further work up in such cases.

\section{Materials and Methods}

This research was descriptive retrospective study. Information was retrieved from clinical and pathological records at Assuit university hospital (considered the largest center of Upper Egypt, receiving cases from all hospitals of Upper Egypt) covering the period in five years from 2009 to 2014. The selected patients were those with chronic unexplained diarrhoea and their colonoscopy findings were normal and submitted to multiple colonscopic biopsies. The inclusion criteria were diarrhoea for at least 6 weeks and normal mucosa without macroscopic changes on colonscopy. The study parameters included age, sex and histopathological findings. The histopathological diagnosis based on the classification of Atlas of Non tumor Pathology Gastrointestinal Diseases [6]: as collagenous colitis defined as chronic 
Citation: Badary DM, Hafez MZ (2017) Role of Mucosal Colonic Biopsy in Patients with Chronic Unexplained Diarrhoea who their Colonoscopy is Normal. Mol Biol 6: 185. doi:10.4172/2168-9547.1000185

Page 2 of 4

inflammation of the mucosa associated with thick subepithelial fibrous layer measuring over $10 \mu \mathrm{m}$, lymphocytic colitis defined as chronic inflammation of the mucosa associated with increased number of intraepithelial lymphocytes in surface epithelium - at least 20 lymphocytes per 100 epithelial cells (average: 25-32), ulcerative colitis defined as diffuse mucosal inflammation with basal plasmacytosis of lamina propria, neutrophilic cryptitis, crypt abcesses, mucosal crypt distortion and mucin depletion, Bilharzial colitis diagnosis based on detection of bilharzial ova which were present in the mucosa or submucosa and were accompanied by inflammatory or fibroblastic response and non-specific inflammation which appears to not matching the criteria for specific inflammation but exceeding the limits of the normal mucosa or reactional.

The biopsy samples have been analyzed in detail under light microscope using hematoxylin- Eosin stain and Masson trichrome stain for collagen. The collected data were submitted to descriptive statistical analysis.

\section{Results}

During a five year period, the clinical records included 300 consecutive patients with chronic unexplained diarrhoea undergoing colonoscopy by one endoscopist, 200 (66.66\%) of who met the inclusion criteria: diarrhoea for at least 6 weeks with normal mucosa on colonscopy. Out of the 200 biopsies considered $68 \%$ of them belong to women and $32 \%$ to men. After a detailed histopathological examination of the samples (Figure 1), $36(18 \%)$ cases were classified as no pathological diagnosis as their biopsy showed normal histopathological examination and $164(82 \%)$ cases yielded specific diagnoses which included $121(73.78 \%)$ cases of non-specific inflammation, $11(6.7 \%)$ cases of collagenous colitis (Figures $2 \mathrm{~A}$ and 2B), 7 (4.26\%) cases of lymphocytic colitis (Figures 2C and 2D), 19 $(11.58 \%)$ cases of ulcerative colitis (Figure 3) and 6 (3.65\%) cases of bilharzial colitis (Figure 3A). The samples were analyzed for age incidence and resulted as collagenous colitis and lymphocytic colitis appeared in the age group range from 25 years to 70 years and the majority were females, ulcerative colitis presented in 40-50 age group, most of patients presented with non-specific inflammation were aged between 30-50 years and all the 6 cases of bilharzial colitis appeared in the 3 rd decade.

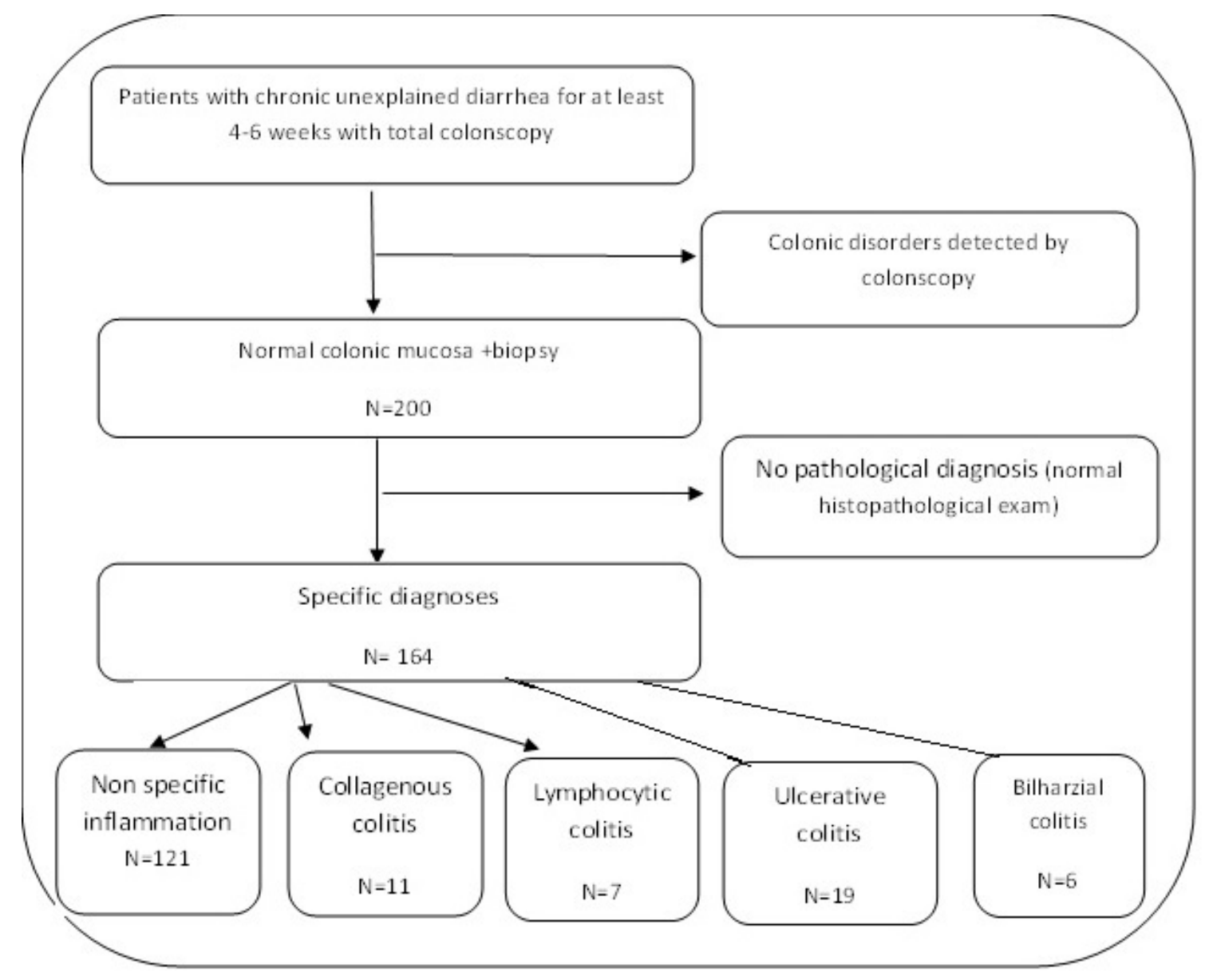

Figure 1: Diagnostic steps of patients with chronic diarrhoea undergoing colonoscopy. 
Citation: Badary DM, Hafez MZ (2017) Role of Mucosal Colonic Biopsy in Patients with Chronic Unexplained Diarrhoea who their Colonoscopy is Normal. Mol Biol 6: 185. doi:10.4172/2168-9547.1000185

Page 3 of 4
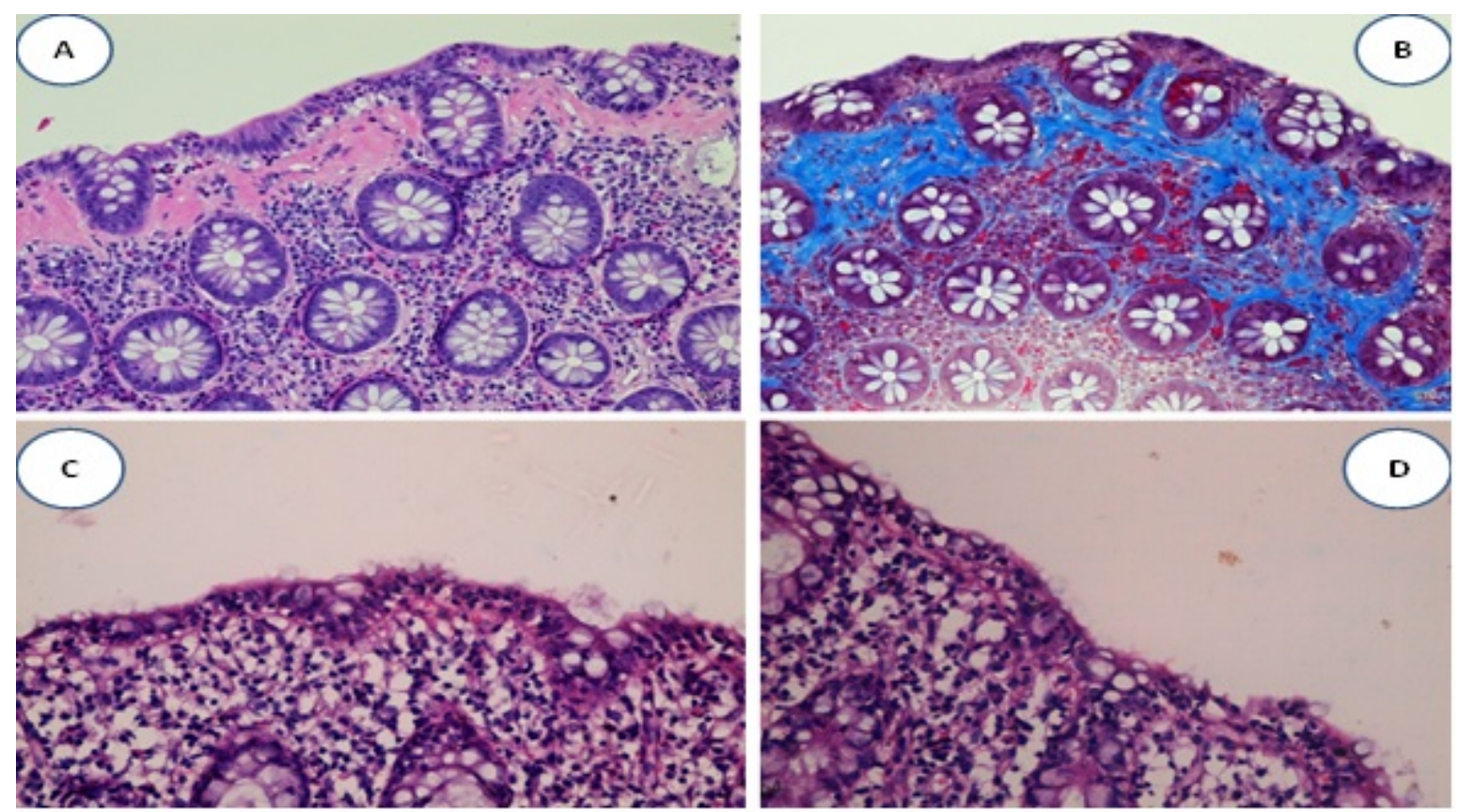

Figure 2: (A) Collagenous colitis; H\&E staining (x200). (B) Collagenous colitis; Masson's trichrome staining (x200). (C) and (D) Lymphocytic colitis with increased intraepithelial lymphocytes; H\&E staining (x400).
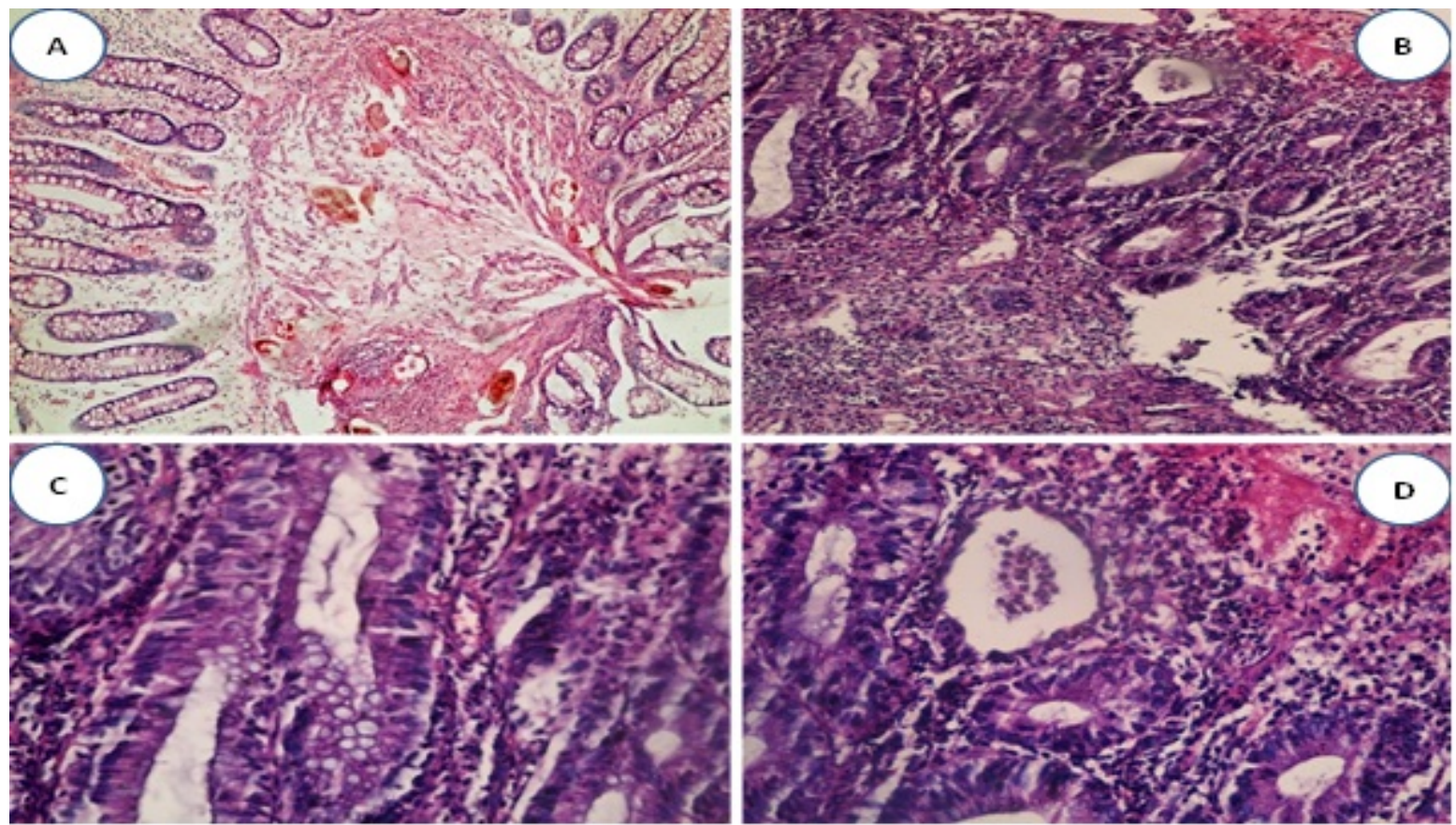

Figure 3: (A) Bilharzial colitis. Multiple ova in the submucosa; H\&E staining (x200). (B) Ulcerative colitis. Diffuse mucosal inflammation with basal plasmacytosis of lamina propria; H\&E staining (x200) (C) Ulcerative colitis. Mucosal crypt distortion and neutrophilic cryptitis; H\&E staining (x400) (D) Ulcerative colitis. Crypt abcesses and mucin depletion; H\&E staining (x400). 


\section{Discussion}

There are many controversies about taking routine biopsy from patients with normal gross colonoscopy. Many physicians prefer performing routine mucosal biopsies to exclude organic causes of GIT symptoms as chronic diarrhoea that can only be diagnosed microscopically even when the endoscopic findings are normal. Some Finding by histopathology may alter the therapeutic decisions by implicating the use of immunosuppressive drugs such as corticosteroid or the need for close clinical follow up [7].

Some authors reported that biopsies should be taken routinely [8] while others argue about the cost-effectiveness of the procedure [9]

The Royal College of pathologists documented that endoscopic biopsy should only be done in patient in the correct clinical setting with a history of persistent watery diarrhoea without blood [4], while the British Society of Gastrointestinal Endoscopy stated in their document Quality and Safety Indicators for endoscopy that biopsies should be performed in all individuals with persistent diarrhoea [5].

Number of biopsies from apparent normal mucosa in patients with chronic unexplained diarrhoea is still controversial although Marshall et al. [9] suggested six biopsies from whole colon in patients with chronic diarrhoea should be sufficient.

Despite the normal colonic appearance in endoscopy of these patients with chronic unexplained diarrhoea in our study, most of the collected biopsies $164(82 \%)$ revealed histopathological changes. This correlates with findings of Kagueyama [10].

Also, Abdelhai et al. found 15 to $40 \%$ of normal endoscopy had histopathological causes of diarrhoea [11].

Also, Lee [7] reported as there was clinically significant histological findings that could alter the theurapeutic decisions in $7.6 \%$ of patients (9/118); two cases of collagenous colitis, one case of lymphocytic colitis, one case of esinophilic enterocolitis, one case of ulcerative colitis and four cases of melanosis coli and [12] studied 308 patients with gastrointestinal complaints as chronic diarrhoea, abdominal pain and other symptoms and normal colonoscopies. They found that there were 30/308 (9.7\%) endoscopically normal cases showed pathological abnormalities. 9/308 (2.9\%) cases of microscopic colitis were detected and of the thirty case with pathological abnormalities, twenty $(66.7 \%)$ case presented by change in the bowel habit.

Reported study has performed mucosal biopsies from normal looking mucosa obtained from 111 chronic diarrhoea patients [9]. They included all patients who had a change in their bowel habits, their data included one case of collagenous colitis and 13 (11.7\%) cases that showed some features of lymphocytic colitis of which the clinical significance was denied by the authors. They thought that these cases should not be considered as trivial or non-specific findings.

In contrast, Rafi et al. found that biopsy of the colon in patients with normal colonscopy had little diagnostic value [2].

In the present study, colonic biopsies enabled us to identify histopathological causes of unexplained chronic diarrhoea in nearly $164(82 \%)$ of a sample of patients whom colonoscopic findings were normal, approximately $43(21.5 \%)$ of them could be treated by an appropriate therapy based on the findings of collagenous colitis, lymphocytic colitis, ulcerative colitis and bilharzial colitis.

Results of histopathological evaluation of specimens from patients with diarrhoea lasting at least 6 weeks displayed a high percentage of specific causes $(82 \%)$, suggesting that biopsies in those patients are very useful for specify the appropriate treatment. As Fine and Schiller reported that serial biopsy helps in establishing a histopathological diagnosis in $15 \%$ of patients with diarrhoea lasting over four weeks [13]. Also, Kagueyama reported that patients with diarrhoea lasting 15-30 days displayed a high incidence of hitological changes [10].

\section{Conclusion}

We conclude that the role of biopsies in chronic diarrhoea patients with grossly normal colon at endoscopy is high as yielding a histological diagnosis in $21.5 \%$ of patients is so benefit to them as they may had modified the therapy received after identification of a specific cause as collagenous colitis, lymphocytic colitis, ulcerative colitis and bilharzial colitis. So Patients should be subject to colonoscopy and biopsy to aid specific diagnoses.

\section{References}

1. Ferreira S (2012) Diarreia crónica. GE Jornal Português de Gastrenterologia 19: 140-142.

2. Rafi UZ Manzar, Mujeeb-Ur-Rehman A (2008) Chronic diarrhea: Large gut causes. Professional Med J Dec 15: 479-485.

3. Nossa F (1999) Colonoscopia diagnóstica e terapêutica. Avaliação das indicações e resultados. Revista Brasileira de Coloproctologia 19: 168-171.

4. Howat A (2005) Histopathology and cytopathology of limited or no clinical value. The Royal College of Pathologists.

5. Valori R, Barton R (2007) BSG quality and safety indicators for endoscopy. JAG: Joint Advisory Group on GI Endoscopy 1-13.

6. Fenoglio-Preiser C (1989) Gastrointestinal pathology an atlas and textraven press. New York.

7. Lee JH (1997) The role of mucosal biopsy in the diagnosis of chronic diarrhea: value of multiple biopsies when colonoscopic finding is normal or nonspecific. Korean J Intern Med 12: 182-187.

8. Prior A, Lessells AM, Whorwell PJ (1987) Is biopsy necessary if colonoscopy is normal? Dig Dis Sci 32: 673-676.

9. Marshall JB, Singh R, Diaz-Arias AA (1995) Chronic, unexplained diarrhea: Are biopsies necessary if colonoscopy is normal? Am J Gastroenterol 90: 372-376.

10. Kagueyama FMN (2014) Importance of biopsies and histological evaluation in patients with chronic diarrhea and normal colonoscopies. Arq Bras Cir Dig (São Paulo) 27: 184-187.

11. Abdelhai AR, Ismail WAF, El-Kashishy KA (2014) The yield of mapping biopsy from apparent endoscopically normal colon and correlation with symptoms. J Gastroenterol Hepatol Res 3: 1168-1172.

12. Elliot VJ, Bateman AC, Green B (2012) The endoscopically normal colon when is mapping biopsy histopathologically justifiable. Frontline Gastroenterol 3: 104-108.

13. Fine KD, Schiller LR (1999) AGA technical review on the evaluation and management of chronic diarrhea. Gastroenterology 116: 1464-1486. 